\title{
A Case of ANCA-associated Large Vessel Vasculitis with Multiple Saccular Aneurysms
}

HIROKAZU SASAKI, MD, Department of Rheumatology, Graduate School of Medical and Dental Sciences, Tokyo Medical and Dental University (TMDU); HAYATO YAMAZAKI, MD, Department of Rheumatology, Graduate School of Medical and Dental Sciences, TMDU; HITOSHI KOHSAKA, MD, PhD, Department of Rheumatology, Graduate School of Medical and Dental Sciences, TMDU, Tokyo, Japan. Address correspondence and reprint requests to Dr. H. Kohsaka, Department of Rheumatology, Graduate School of Medical and Dental Sciences, Tokyo Medical and Dental University, 1-5-45 Yushima, Bunkyo-ku, Tokyo, Japan. E-mail address: kohsaka.rheu@tmd.ac.jp. J Rheumatol 2016;43:179; doi:10.3899/jrheum.150435

Aortitis is rare, but can occur in antineutrophil cytoplasm autoantibody (ANCA)-associated vasculitis (AAV) ${ }^{1}$. A high titer of proteinase 3 (PR3)-ANCA and renal involvement also suggest AAV. Giant cell arteritis, Behçet disease, and IgG4-related disease may account for multiple saccular aneurysms $2,3,4$.

A 66-year-old man presenting with persistent fever, low back pain, and newly developed hypertension was referred to our hospital. On admission, laboratory data showed elevated C-reactive protein level $(6.44 \mathrm{mg} / \mathrm{dl})$ and a high titer of PR3-ANCA (53.0 IU/ml), while other specific antibodies were negative. Urinalysis revealed microhematuria, proteinuria $(0.91 \mathrm{~g} / \mathrm{gCre})$, and elevated $\mathrm{N}$-acetyl- $\beta$-D-glucosaminidase level (20 IU/ml), which indicated renal impairment. Because serum creatinine did not increase, kidney biopsy was not performed. Contrast-enhanced computer tomography depicted the wall thickening of multiple saccular aneurysms in the thoracic and abdominal aorta (Figure 1) and of the stenosed renal artery.

Positive PR3-ANCA, urinary abnormalities, and large vessel lesions were diagnosed as AAV. Clinical remission and normalization of PR3-ANCA value were attained by treatment with prednisolone (PSL) and intravenous cyclophosphamide. After tapering of PSL, aortic wall thickening and microhematuria relapsed, accompanied by an increase of PR3-ANCA. Retreating with high-dose PSL and rituximab led to remission again, and allowed aortic replacement surgery to be performed. Histopathological examination of the resected vessel wall revealed the degenerated media and ruptured external elastic lamina without atherosclerosis, indicating healed aortitis.

A high titer of PR3-ANCA was highly specific for AAV 5 and no manifestations of this case were suggestive of these disorders. This is the rare case of ANCA-associated large vessel vasculitis, forming multiple saccular aneurysms.

\section{REFERENCES}

1. Chirinos JA, Tamariz LJ, Lopes G, Del Carpio F, Zhang X, Milikowski C, et al. Large vessel involvement in ANCA-associated vasculitides: report of a case and review of the literature. Clin Rheumatol 2004;23:152-9.

2. García-Martínez A, Arguis P, Prieto-González S, Espígol-Frigolé G, Alba MA, Butjosa M, et al. Prospective long term follow-up of a cohort of patients with giant cell arteritis screened for aortic structural damage (aneurysm or dilatation). Ann Rheum Dis 2014;73:1826-32.

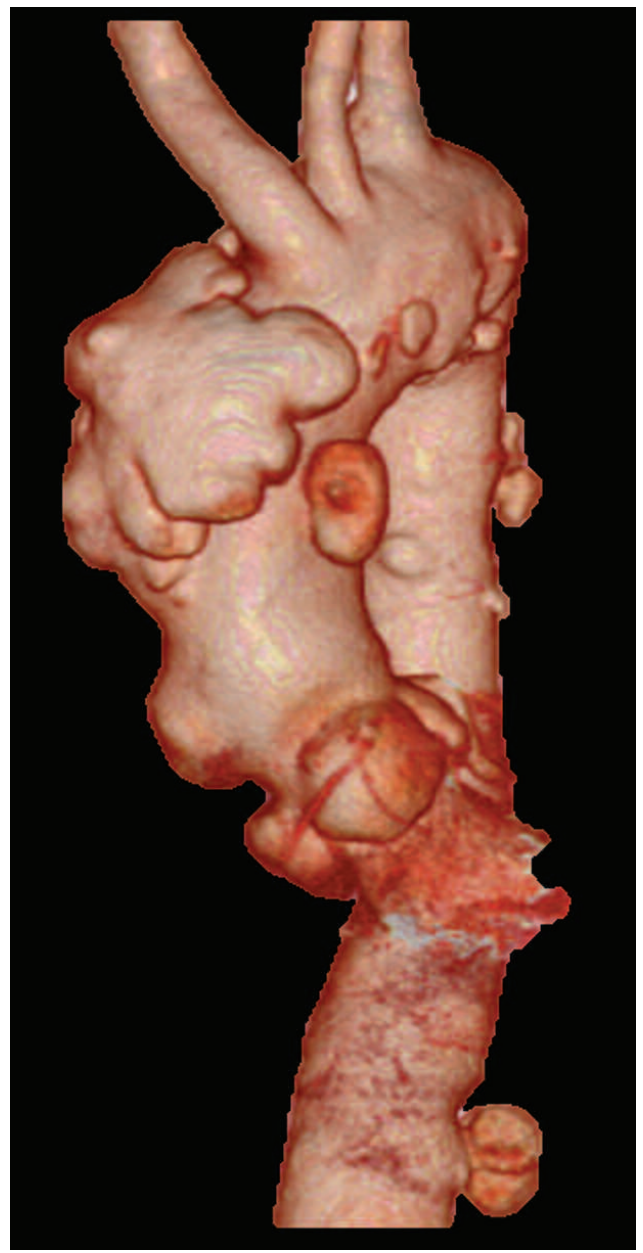

Figure 1.3-D computed tomography angiography of the aorta on admission shows multiple saccular aneurysms.

3. Saadoun D, Asli B, Wechsler B, Houman H, Geri G, Desseaux K, et al. Long-term outcome of arterial lesions in Behçet disease: a series of 101 patients. Medicine 2012;91:18-24.

4. Stone JR. Aortitis, periaortitis, and retroperitoneal fibrosis, as manifestations of IgG4-related systemic disease. Curr Opin Rheumatol 2011;23:88-94.

5. Hagen EC, Daha MR, Hermans J, Andrassy K, Csernok E, Gaskin $\mathrm{G}$, et al. Diagnostic value of standardized assays for anti-neutrophil cytoplasmic antibodies in idiopathic systemic vasculitis. EC/BCR Project for ANCA Assay Standardization. Kidney Int 1998; 53:743-53. 